\title{
Cardinal Henri De Lubac, Jacques Maritain, Correspondance et rencontres. Tome L.
}

Préface par le cardinal Philippe Barbarin

Notes bibliographiques, index, texte établi, annoté et présenté par JeanMichel Garrigues et René Mougel

Paris, Les Éditions du Cerf, coll. « Euvres du Cardinal Henri de Lubac et Études Lubaciennes », 50, 2012, 144 p.

\section{Paul Airiau}

\section{(2) OpenEdition} Journals

\section{Édition électronique}

URL : http://journals.openedition.org/assr/25548

DOI : $10.4000 /$ assr. 25548

ISSN : $1777-5825$

Éditeur

Éditions de l'EHESS

Édition imprimée

Date de publication : 30 décembre 2013

Pagination : 238

ISSN : 0335-5985

Référence électronique

Paul Airiau, "Cardinal Henri De Lubac, Jacques Maritain, Correspondance et rencontres. Tome L. », Archives de sciences sociales des religions [En ligne], 164 | 2013, mis en ligne le 06 mars 2014, consulté le 21 septembre 2020. URL : http://journals.openedition.org/assr/25548 ; DOI : https://doi.org/ $10.4000 /$ assr. 25548

Ce document a été généré automatiquement le 21 septembre 2020.

(C) Archives de sciences sociales des religions 


\section{Cardinal Henri De Lubac, Jacques Maritain, Correspondance et rencontres. Tome L.}

Préface par le cardinal Philippe Barbarin

Notes bibliographiques, index, texte établi, annoté et présenté par JeanMichel Garrigues et René Mougel

Paris, Les Éditions du Cerf, coll. « Euvres du Cardinal Henri de Lubac et Études Lubaciennes ", 50, 2012,144 p.

Paul Airiau

\section{RÉFÉRENCE}

Cardinal Henri De Lubac, Jacques Maritain, Correspondance et rencontres. Tome L, Préface par le cardinal Philippe Barbarin, Notes bibliographiques, index, texte établi, annoté et présenté par Jean-Michel Garrigues et René Mougel, Paris, Les Éditions du Cerf, coll. « CEuvres du Cardinal Henri de Lubac et Études Lubaciennes », 50, 2012, 144 p. 
1 La publication des œuvres complètes du cardinal Henri de Lubac suit son bonhomme de chemin. Elle prend ici la forme de l'édition de 17 lettres échangées entre le jésuite et Jacques Maritain (p. 43-80). Celle-ci est encadrée en ouverture d'une courte préface du cardinal archevêque de Lyon Philippe Barbarin (p.9-12) et d'une longue introduction du fr. Jean-Miguel Garrigues, "Jacques Maritain et Henri de Lubac: distance, convergences et rapprochement » (p.13-42, parue précédemment dans la Revue théologique des Bernardins, $\mathrm{n}^{\circ} 4$, février 2012, p. 65-96, http://www.revuedesbernardins.com/ spip.php?article334). Elle est fermée par une « Note sur les renvois à l'œuvre de J. Maritain dans l'ouvrage Sur les chemins de Dieu » (p. 83-84); la reprise (p. 85-114)

Cardinal Henri de Lubac Jacques Maritain

\section{Correspondance et rencontres}

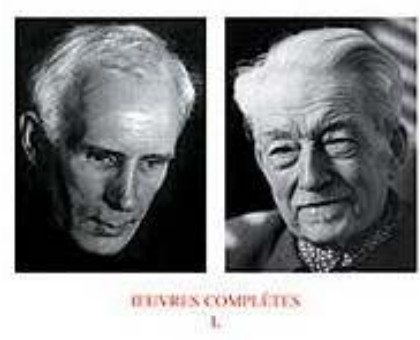

cerf d'une communication de René Mougel, «La position de J. Maritain à l'égard de Surnaturel: le péché de l'ange, ou esprit et liberté » (donnée au colloque «Surnaturel, une controverse au cœur du thomisme au $\mathrm{xx}^{\mathrm{e}}$ siècle » organisé par l'Institut SaintThomas-d'Aquin de Toulouse les 26-27 mai 2000, publiée dans la Revue thomiste, 2001/1-2, p. 73-98) ; et un inédit de Florian Michel, « Jacques Maritain et Henri de Lubac en dialogue sur les traductions liturgiques (1965-1970)»(p.115-130 - signalons au passage l'intérêt des publications de F. Michel consacrées à la liturgie catholique au moment de sa réforme duovaticane : il a eu accès à des archives peu ouvertes...).

2 Soit donc 17 lettres, 17 petites lettres, correspondant à 35 pages (dont une partie parfois non négligeable d'annotation), et une introduction et trois études totalisant en tout 78 pages. Faut-il le dire? On a l'impression d'entrer, en pleine histoire contemporaine, dans des pratiques dont on pensait qu'elles étaient réservées surtout aux antiquisants et autres médiévistes, qui, se fondant sur un faible voire minime matériau, réussissent à produire des analyses défiant toute imagination de contemporanéiste, et avec une pertinence toujours stupéfiante - enfin, nuançons : les publications de sources même en histoire contemporaine nécessitent toujours un appareil critique, qui peut parfois être abondant, et dont les introductions peuvent atteindre des proportions conséquentes. Aboutit-on pourtant au même résultat avec ce qui reste essentiellement des félicitations, des remerciements, des mots aimables, des manifestations d'accord ou de proximité, avec seulement deux lettres dépassant les deux pages imprimées au format $15 \times 20 \mathrm{~cm}$ ? La chose reste à voir.

D'emblée, relevons que les deux annexes s'attachent à deux points qui ne sont pas forcément l'essentiel de la correspondance. R. Mougel met en valeur le rapprochement entre Maritain et Lubac sur des questions de théologie et d'anthropologies fondamentales, la théorie de la nature pure, le désir naturel de Dieu, la peccabilité angélique, points qui relèvent de la querelle de la «nouvelle théologie » qui déchira 
violemment le catholicisme français en 1946-1950, alors que seules deux lettres évoquent la question. F. Michel, partant d'un échange entre Lubac et Maritain dans la première moitié d'août 1969 évoquant la question des traductions en français des textes liturgiques, présente en fait plus largement les positions de chacun des deux hommes avant de conclure sur leur rapprochement sur cette question et le sens que ce fait peut avoir dans l'histoire du catholicisme français - on y reviendra.

Pour aborder l'ouvrage, on conseillera de débuter par les lettres. Leur chronologie n'est pas insignifiante, quoique le point ne soit pas assez mis en avant. Six lettres sont échangées de 1937 à 1963, puis onze de 1967 à 1972. Les six premières marquent des rapprochements entre un des maîtres du thomisme et un ténor du "tiers parti réformateur " (tel qu'étudié par Étienne Fouilloux dans Une Église en quête de liberté. La pensée catholique française entre modernisme et Vatican II, 1914-1962, Paris, Desclée de Brouwer, 1998, compte rendu ASSR, 106-26), à partir du moment où Maritain est perçu comme un des théoriciens d'une action catholique ne visant pas la restauration d'une chrétienté, ce qui lui vaut des attaques de plus en plus fortes, notamment de la jésuite et romaine Civiltà cattolica en 1956. Lubac et Maritain se retrouvent ainsi dans une commune situation, d'où l'expression d'une forme de solidarité et d'accord. Les victimes possibles ou désignées de mes censeurs d'autrefois sont potentiellement mes amis, telle pourrait être l'idée... Plus importante est la lettre de 1961. Lubac y affirme sa satisfaction de se trouver d'accord avec Maritain sur une thèse essentielle de Surnaturel, soit la peccabilité de l'ange, que le dernier vient de soutenir dans un volume collectif (Le péché de l'ange) qu'il a fait expédier au premier. Ou plutôt, il faudrait comprendre que Lubac se réjouit en fait que Maritain se rallie aux positions que le premier avait défendues dans Surnaturel en 1946. Mais Lubac marque malgré tout les limites d'un possible rapprochement : "Maintenant, ces questions sont bien loin de moi. [...] il y a bien longtemps que je n'ai plus étudié la question, et maintenant toutes sortes d'autres petites besognes, accordées à mon âge et à mes forces, m'en détournent » (p. 54). À croire que les 24 ans de moins que Lubac affiche au compteur et son état de santé l'empêchent de traiter une question qu'il reprendra ultérieurement, qui a été fondamentale dans son itinéraire intellectuel et pour laquelle il a été violemment attaqué au sortir de la guerre... Bref, comme fin de non-recevoir, il paraît difficile de faire plus jésuite. La question revient d'ailleurs dans la lettre suivante, en 1963, lorsque Lubac se félicite de nouveau, après que Maritain lui a fait de nouveau parvenir un ouvrage, Dieu et la permission du mal, que le philosophe tienne la peccabilité de l'ange.

5 Si convergence il y a, elle demeure réellement limitée. À moins qu'il ne faille absolument se rallier à l'idée sous-tendue par R. Mougel et le p. Garrigues, que, dans l'équilibre général de la cosmologie thomiste et de l'anthropologie chrétienne, la possibilité du péché de l'esprit créé est un point fondamental. Certes, Lubac a passé toute une partie de Surnaturel à l'établir, mais la controverse s'est essentiellement développée sur d'autres points de l'ouvrage. Faudrait-il donc croire que l'essentiel peut se dire en peu de mots, et que les remarques dubitatives que l'on a émises plus haut ne sont pas pertinentes? À défaut d'être certain, c'est au moins possible. Mais, malgré tout, cela laisse au moins de côté toute la question de la nature pure, qui fut au cœur des débats entre Lubac et ses détracteurs - et que Pie XII traita dans Humani generis, la peccabilité de l'ange n'ayant pas cet honneur.

6 En matière de rencontre, le dossier est donc maigre avant 1964. Les choses changent après Vatican II. Maritain et Lubac partagent les mêmes inquiétudes sur l'« esprit du 
concile ", l'écrivent, se découvrent l'écrivant et finalement se l'écrivent. Cependant, il demeure entre eux des divergences, même si leur expression va aller en s'atténuant, et dans des formes qui se font plus délicates. Deux points entraînent des désaccords, l'un mineur, en 1969, l'autre majeur, en 1967. Le premier est la traduction de l'article du Credo «Et in unam sanctam catholicam et apostolicam Ecclesiam ». Lubac rejette la version française, "Je crois en l'Église ", qui, pour lui, met la foi en Dieu et en l'Église sur le même plan et casse la structure trinitaire de la profession de foi. Maritain est moins critique, estimant que la "grammaire a une majesté très relative" (p.69), même s'il comprend les inquiétudes théologiques exprimées par le jésuite. Le second point de désaccord concerne le P. Teilhard de Chardin. Maritain met en cause son influence dans la manifestation brutale d'une « apostasie immanente » dans le catholicisme, comme il l'affirme dans Le Paysan de la Garonne. Mais, sur Teilhard, Lubac demeure intraitable (il y aura sans doute un jour à s'interroger sur le teilhardisme de Lubac, et il serait opportun que ce ne fût ni un teilhardien, ni un lubacien, ni un antiteilhardien, qui s'attache à cette question). Cependant, Lubac met les formes dans la manifestation de son désaccord: rejet d'un teilhardisme frelaté, regret que Teilhard ne soit pas compris par le cardinal Charles Journet et Maritain, mais louanges au Paysan de la Garonne.

7 Bref, il n'est pas sûr que ces lettres soient, autant que les commentateurs l'écrivent volontiers dans cette édition, des pierres milliaires pour marquer la convergence entre Lubac et Maritain. L'accord sur la peccabilité de l'ange est acté en 1961-1963, mais aucune conséquence n'en est largement tirée. La défiance perdure, et un certain nombre de données parsemant le texte et les notes de l'introduction et des études le montrent : regard fort critique de Maritain sur l'équipe présidant à la fondation de Concilium en 1965, à laquelle appartient Lubac (note 3 p.15-16); dévalorisation de Maritain par Lubac dans les Mémoires sur l'occasion de mes écrits (1976, 1981, publié en 1989) et l'annexe de l'édition des Lettres de monsieur Étienne Gilson au P. de Lubac (1986) (p. 15 et note 1 p. 111). Cependant, la deuxième époque de la correspondance montre au moins que, face à une situation imprévue, un brutal bouleversement intellectuel, Maritain et Lubac se retrouvent subitement sur une ligne commune. À ce titre, la finale de l'étude de F. Michel prend toute son importance, lorsqu'il pointe la recomposition en cours dans les années 1965-1970, recomposition qu'il faut observer finement pour ne pas durcir les positions ni les caricaturer en fonction de ce qui sera plus tard (p. 129).

Oui, il faut le poser fortement: les années 1965-1970 furent celles de la découverte réelle par deux des courants du tiers parti réformateur, le courant de la «nouvelle théologie » et les maritainiens en rupture avec le thomisme romain et éloigné du thomisme historicisé du Saulchoir, qu'ils avaient des points communs et qu'ils devaient se rapprocher s'ils voulaient que subsistât ce à quoi ils tenaient fondamentalement pardelà leurs désaccords théologiques et pastoraux. La manifestation brutale d'autres positions transforme le paysage théologique au point de faire passer pour de moindre importance les divergences antérieures, car, en fait, elles se situaient dans un univers théologique commun qui n'est plus partagé avec les autres manières de faire de la théologie. D'une certaine manière, le thomisme maritainien et la théologie lubacienne d'inspiration patristique et blondélienne sont bien deux paradigmes, confrontés donc à une logique d'incommunicabilité. Mais, avec les transformations intellectuelles, ils découvrent qu'ils partagent en fait un métaparadigme, mais que celui-ci ne peut communiquer (dans une mesure qu'il faudrait aussi peser) avec les paradigmes alternatifs qui se développent. Sur ces questions, des travaux ont déjà été menés en français, en particulier par Étienne Fouilloux, qui fut un précurseur pour poser les 
bases de la compréhension de la situation post-conciliaire, Michel Fourcarde, et, du point de vue du théologien engagé, Christoph Theobald, sans oublier Un nouvel âge de la théologie? 1965-1980. Colloque de Montpellier, juin 2007, Paris, Karthala, coll. Signes des Temps, 2009 (recension par Sabine Rousseau, ASSR, 152). On ne peut que souhaiter que ces recherches se poursuivent.

De manière fort satisfaisante pour l'analyste, l'introduction du fr. Garrigues et l'étude de R. Mougel confirment bien que s'est réalisée la recomposition voyant la convergence entre maritainisme et lubacisme. Avec leur vocabulaire et leur cadre d'analyse, ils affirment qu'elle a eu lieu, prenant comme exemple canonique Fides et Ratio de JeanPaul II, fruit notamment du travail conjoint du p. Georges Cottier, héritier du cardinal Journet, et d'héritiers de la "nouvelle théologie» (note 2 p. 41 - voir aussi la reprise par le cardinal Barbarin p. 11). Cependant, faut-il le dire ? La divergence paraît malgré tout perdurer, dans chacune des deux branches ayant participé à la recomposition. Toute l'étude du p. Garrigues vise à montrer que, in fine, Maritain a fini par trouver dans Thomas d'Aquin des éléments pour converger avec Lubac qui avait foncièrement raison sur la querelle du surnaturel. L'affirmation renouvelée de la page 36 est trop insistante pour ne pas manifester que le p. Garrigues tend à préférer théologiquement le jésuite au laïc : «Maritain va converger de plus en plus avec Lubac dans le souci de conduire l'homme moderne à la vérité en la lui faisant chercher librement. [...] Nous voudrions relever les étapes successives du chemin par lequel Maritain a convergé vers l'intuition maîtresse de Lubac sur le surnaturel. »Cependant, la finale de l'introduction est plus mesurée : c'est une convergence commune qui s'observe. Chez R. Mougel, la position est différente. Maritain y a un rôle plus fort : «[...] la philosophie thomiste de Jacques Maritain a apporté à la grande intuition théologique - et blondélienne aussi du P. de Lubac dans son combat contre le système du parallélisme nature-surnature [...] un puissant renfort puisqu'elle pulvérisait, en métaphysique, ce système, sur le double sujet de la peccabilité naturelle de l'esprit créé et de la vie unitaire de la Fin ultime dans sa moralité. Leur concours laissait ainsi la place pour une grande théologie du surnaturel, trinitaire et christique, qui retrouverait chez saint Thomas ses principes [...], libérée des embarras faussement "métaphysiques" que des théologiens avaient conçus [...]» (p. 113-114). Ainsi, l'importance de l'accord paraît donc finalement limitée, et c'est peut-être avoir beaucoup écrit pour aboutir, in fine, à un résultat relativement réduit. Mais n'est-ce pas souvent, aussi, le propre de la vie intellectuelle?

À moins qu'il ne faille ouvrir une autre piste d'interprétation de cette proclamation incessante d'une convergence. Car, sur tout ceci, ces tensions, recompositions, désaccords, retrouvailles partielles, convergences incomplètes, la préface propose de jeter désormais un regard de charité en argumentant à partir de l'existence de polarité dans la pensée (en utilisant Erich Przywara et son travail sur l'analogie). Le cardinal Barbarin est ici, indéniablement dans son rôle. Et il l'est peut-être encore plus qu'il ne pourrait le penser. Car il théorise, pour les théologiens au pouvoir dans les instances hiérarchiques du catholicisme contemporain, au moins ceux de la Curie et ceux qui s'inscrivent dans leur paradigme, la validité de la recomposition théologique qu'ils ont produite entre 1965 et 1975 . Ces dix-sept lettres auraient donc une fonction sociale très nette, qu'on ne saurait occulter. Mais il est vrai que la mesure épiscopale et théologique est spécifique, et qu'elle n'est pas celle d'un recenseur. Celui-ci pourra alors au moins conclure, dans une dernière pirouette, qu'il n'est pas négligeable, d'un point de vue 
historique, qu'un prince de l'Église, après Vatican II, justifie, dans une mesure relative, la pluralité des paradigmes théologiques. 